\title{
On the Uniqueness of the Equilibrium State for Ising Spin Systems
}

\author{
Joel L. LeBowitz * \\ Belfer Graduate School of Science, Yeshiva University, New York, N.Y. \\ ANDERS MARTIN-LöF \\ Rockefeller University, New York, N.Y. \\ Received November 10, 1971
}

\begin{abstract}
We show that for an Ising spin system of arbitrary spin with a ferromagnetic pair interaction and a "periodic" external magnetic field there is a unique equilibrium state if and only if the magnetization is continuous with respect to a uniform change in the external field. Hence, if the critical temperature $T_{c}$ is defined as the temperature where the spontaneous magnetization (which is a non-increasing function of the temperature) becomes positive, then the equilibrium state is unique for $T>T_{c}$ and is non-unique for $T<T_{c}$ (when the external field is zero). This implies that the correlation functions have a cluster property for $T>T_{c}$.

We also show that for an anti-ferromagnet consisting of two sublattices there is a unique equilibrium state if and only if the staggered magnetization is continuous with respect to a change in the staggered field.
\end{abstract}

\section{Introduction}

We consider an Ising spin system with a ferromagnetic pair interaction in a finite box $\Lambda$ on a $d$-dimensional lattice $\mathbb{Z}^{d}$, i.e. at each point $p$ of the lattice there is a spin $\sigma_{p}= \pm 1$, and the conditional probability of a spin configuration in the box given a configuration outside it is proportional to

$\exp \left(-E_{A}\right)=\exp \left(\frac{1}{2} \sum_{p \neq q \in A} J_{p-q} \sigma_{p} \sigma_{q}+\sum_{p \in A} \sigma_{p}\left(H_{p}+h+\sum_{q \notin A} J_{p-q} \sigma_{q}\right)\right)$.

$J_{p}=J_{-p} \geqq 0$ is the pair interaction, $\sum_{p \in \mathbb{Z}^{d}} J_{p}<\infty$, and $H_{p}+h$ an external magnetic field. The reciprocal temperature $\beta$ has been included in the Hamiltonian. The external field consists of a uniform part $h$ and a periodic part $H_{p}$, i.e. $H_{p}=H_{p+g}$ when $g$ is contained in some subgroup $G$ of $\mathbb{Z}^{d}$. A boundary condition for the box $\Lambda$ is specified by giving a probability distribution $b_{\Lambda}(d \sigma)$ for the configurations outside $\Lambda$.

The (equilibrium) state of the system in $\Lambda$ is the probability distribution for configurations in $\Lambda$ defined by (1) together with $b_{A}$ or equivalently

\footnotetext{
* Supported in part by U.S.A.F.O.S.R. under contract F 44620-71-C-0013, P001.
} 
by the family of correlation functions

$$
\left\langle\sigma_{A}\right\rangle_{H, h, A, b_{A}} \equiv\left\langle\prod_{p \in A} \sigma_{p}\right\rangle_{H, h, \Lambda, b_{A}} \text { for } A \subseteq \Lambda .
$$

An equilibrium state of the infinite system ${ }^{1}$ is defined to be a family of correlation functions $\left\langle\sigma_{A}\right\rangle_{H, h, b}$ for the finite subsets $A$ of $\mathbb{Z}^{d}$ obtained as the limit of correlation functions for a sequence of finite boxes with some boundary conditions:

$$
\left\langle\sigma_{A}\right\rangle_{H, h, b}=\lim _{A \rightarrow \infty}\left\langle\sigma_{A}\right\rangle_{H, h, A, b_{A}} \text { for all finite } A .
$$

We say that a sequence of boxes tends to infinity if they increase and if every finite subset of $\mathbb{Z}^{d}$ is contained in some $\Lambda$ of the sequence, and if $\left|\Lambda_{l}\right| /|\Lambda| \rightarrow 0$ for any $l \geqq 0$, where $\Lambda_{l}$ is the set of points in $\Lambda$ with distance at most $l$ from its complement, and $|\Lambda|$ denotes the number of points in $A$ (van Hove convergence). The letter $b$ indicates some arbitrary boundary condition, and $b_{A}=+$ or - will indicate the boundary conditions defined by putting all spins outside $\Lambda=+1$ or -1 . The statement, $\left\langle\sigma_{A}\right\rangle_{0, h, b}$ does not depend on the boundary condition is equivalent to the statement that the equilibrium state is unique, Dobrushin [1].

For a finite system the average magnetization $m\left(H, h, \Lambda, b_{A}\right)$ $\equiv\left\langle\frac{1}{|\Lambda|} \sum_{p \in \Lambda} \sigma_{p}\right\rangle_{H, h, \Lambda, b_{A}}$ is equal to $\frac{\partial F\left(H, h, \Lambda, b_{A}\right)}{\partial h}$, where $F\left(H, h, \Lambda, b_{A}\right)$ $=\frac{1}{|\Lambda|} \log \left(\sum_{\sigma} e^{-E_{\Lambda}(\sigma)}\right)$ is proportional to the free energy. Since $F\left(H, h, \Lambda, b_{A}\right)$ is a convex function of $h$ and it has a limit, $F(H, h)$, which is independent of the boundary condition for the periodic Hamiltonians we consider, [9], the latter is also a convex function of $h$, and thus

$$
m(H, h, b) \equiv \lim _{\Lambda \rightarrow \infty} m\left(H, h, \Lambda, b_{\Lambda}\right)=\frac{\partial F(H, h)}{\partial h}
$$

for any boundary condition whenever this derivative exists. It follows from the convexity of $F(H, h)$ that the right and left derivatives $\frac{\partial F(H, h)}{\partial h^{ \pm}}$ always exist and are right and left continuous respectively, and that they differ at most at a denumerable set of $h$-values.

When $H_{p}=0, F(0, h)$ is analytic in $h$ for $h \neq 0$, Lee and Yang [9]. The spontaneous magnetization $m^{*}$ is defined, for $H_{p}=0$, as the right derivative of $F(0, h)$ at $h=0$,

$$
m^{*}=\lim _{h \rightarrow 0+} \frac{\partial F(0, h)}{\partial h} .
$$

\footnotetext{
${ }^{1}$ For details see Dobrushin [1], Lanford and Ruelle [6] and Ruelle [10].
} 


\section{Main Theorems}

Our results can now be formulated as follows:

Theorem 1. There is a unique equilibrium state for the infinite system, i.e. the $\left\langle\sigma_{A}\right\rangle_{H, h, b}$ do not depend on the boundary condition and have the same invariance (periodicity) as the Hamiltonian if and only if $\frac{\partial F(H, h)}{\partial h}$ exists. This happens if and only if $m(H, h, b)$ is independent of the boundary conditions.

When $H_{p}=0, h \neq 0$, Theorem 1 combined with the Lee-Yang Theorem [9], assures the existence of a unique equilibrium state. This has also been proven recently by Ruelle [11], using similar methods (see comment later). For the case with no external field $H_{p}=0, h=0$ we get:

Theorem 2. When the external field is zero there is a unique equilibrium state for the infinite system if and only if $m^{*}=0$. This happens if and only if $\left\langle\sigma_{p}\right\rangle_{0,0, b}=0$ for all boundary conditions.

For any partitioning of $\mathbb{Z}^{d}$ into two disjoint congruent sublattices $L_{0}$ and $L_{1}$ such that $L_{0}$ is a subgroup of $\mathbb{Z}^{d}$ and $L_{1}$ its coset we can extend our result to the anti-ferromagnetic spin system "adjoint" to the ferromagnetic system we have been considering up to now. The adjoint system is obtained as follows. Call the spin variables of the adjoint system $\hat{\sigma}_{p}= \pm 1$ and let its interaction be $\hat{J}_{p-q}=J_{p-q} \geqq 0$ if $p$ and $q$ are contained in the same sublattice and $\hat{J}_{p-q}=-J_{p-q} \leqq 0$ otherwise, and let the external fields be $\hat{h}_{p}=-H_{p}, \hat{H}_{p}=-h$ if $p \in L_{0}$ and $\hat{h}_{p}=H_{p}, \hat{H}_{p}=h$ if $p \in L_{1} . \hat{H}_{p}$ is then a "staggered field" and $\hat{h}_{p}$ is a uniform field if $H_{p}$ was a "staggered field". (The fact that $L_{0}+L_{0}=L_{0}, L_{0}+L_{1}=L_{1}$ and $L_{1}+L_{1}=L_{1}$, which is easily verified, guarantees that $\hat{J}_{p-q}$ as defined above is actually a translation invariant pair interaction and that $H_{p}$ is periodic, $H_{p+g}=H_{p}$ for $g \in L_{0}$, if $\hat{h}_{p}$ is uniform.) We call $\hat{F}(\hat{H}, \hat{h})$ the free energy of the anti-ferromagnet. By the transformation $\hat{\sigma}_{p}=-\sigma_{p}$ for $p \in L_{0}, \hat{\sigma}_{p}=\sigma_{p}$ for $p \in L_{1}$ this system is changed into the ferromagnet we have been studying and $\hat{F}(\hat{H}, \hat{h})=F(H, h)$. We then have the following result:

Theorem 3. For the anti-ferromagnet described above there is a unique equilibrium state if and only if the "staggered magnetization"

$$
m^{s}(\hat{H}, \hat{h}, b) \equiv \lim _{\Lambda \rightarrow \infty}\left\langle\frac{1}{|\Lambda|} \sum_{p \in L_{1}} \hat{\sigma}_{p}-\sum_{p \in L_{0}} \hat{\sigma}_{p}\right\rangle_{\hat{H}, \hat{h}, \Lambda, b_{\Lambda}}
$$

does not depend on the boundary conditions, which happens if and only if $\frac{\partial \hat{F}(\hat{H}, \hat{h})}{\partial h}$ exists. 
The proofs of Theorem 1-3 follow from the following lemmas, which concern the lattice gas associated to the spin system defined by the "occupation numbers" $\varrho_{p}=\frac{1}{2}\left(1+\sigma_{p}\right)$. Their proofs depend on the recently derived inequalities by Fortuin, Ginibre, Kasteleyn (F. G. K.) [2], which say that if $f$ is a function of the $\varrho_{p}$ in a finite box $A$ which is increasing in each variable separately then $\langle f\rangle$ is an increasing function of the interactions and the external fields at the lattice points if the pair interactions are ferromagnetic and the fields are of arbitrary signs. Because $\sigma_{A}$ is a finite linear combination of the $\varrho_{B}$ for $B \cong A$, and conversely, the uniqueness of the state of the spin system is equivalent to that of the lattice gas.

Lemma 1. $\left\langle\varrho_{A}\right\rangle_{H, h, \pm}=\lim _{A \rightarrow \infty}\left\langle\varrho_{A}\right\rangle_{H, h, A, \pm}$ exist and have the same symmetry as the Hamiltonian, $\left\langle\varrho_{A+g}\right\rangle_{H, h, \pm}=\left\langle\varrho_{A}\right\rangle_{H, h, \pm}$ for $g \in G$. In addition,

$$
\begin{aligned}
\lim _{h^{\prime} \rightarrow h^{+}}\left\langle\varrho_{A}\right\rangle_{H, h^{\prime},+} & =\left\langle\varrho_{A}\right\rangle_{H, h,+}, \\
\lim _{h^{\prime} \rightarrow h^{-}}\left\langle\varrho_{A}\right\rangle_{H, h^{\prime},-} & =\left\langle\varrho_{A}\right\rangle_{H, h,-},
\end{aligned}
$$

Proof. (Some of the ideas used in the following proof are due to at least two authors, Griffiths and Lieb: private communications.) The F.G.K. result applies to $f=\varrho_{A}$ because this is an increasing function of the $\varrho_{p}$. This implies that $\left\langle\varrho_{A}\right\rangle_{H, h, \Lambda^{\prime},+} \leqq\left\langle\varrho_{A}\right\rangle_{H, h, \Lambda,+}$ if $\Lambda^{\prime} \supseteqq \Lambda$, because the latter can be obtained from the former by letting an infinite positive magnetic field act on the sites in $\Lambda^{\prime} \backslash \Lambda$. Hence $\left\langle\varrho_{A}\right\rangle_{H, h,+}=\lim _{\Lambda \rightarrow \infty}\left\langle\varrho_{A}\right\rangle_{H, h, A,+}$ exists and $\left\langle\varrho_{A}\right\rangle_{H, h,+} \leqq\left\langle\varrho_{A}\right\rangle_{H, h, A,+}$. Thus also

$$
\lim _{h^{\prime} \rightarrow h^{+}}\left\langle\varrho_{A}\right\rangle_{H, h^{\prime},+} \leqq \lim _{h^{\prime} \rightarrow h^{+}}\left\langle\varrho_{A}\right\rangle_{H, h^{\prime}, A,+}=\left\langle\varrho_{A}\right\rangle_{H, h, A,+},
$$

and letting $\Lambda \rightarrow \infty$ we see that $\lim _{h^{\prime} \rightarrow h^{+}}\left\langle\varrho_{A}\right\rangle_{H, h^{\prime},+} \leqq\left\langle\varrho_{A}\right\rangle_{H, h,+}$. But F.G.K. also tell us that $\left\langle\varrho_{A}\right\rangle_{H, h,+} \leqq\left\langle\varrho_{A}\right\rangle_{H, h^{\prime},+}$ when $h \leqq h^{\prime}$, so

$$
\left\langle\varrho_{A}\right\rangle_{H, h,+} \leqq \lim _{h^{\prime} \rightarrow h+}\left\langle\varrho_{A}\right\rangle_{H, h^{\prime},+}
$$

and (4) is proved. (5) is proved analogously.

The symmetry of the $\left\langle\varrho_{A}\right\rangle_{H, h, \pm}$ follows from the uniqueness of taking the limit $A \rightarrow \infty$ with these boundary conditions which is again a consequence of the F.G.K. inequalities.

\section{Lemma 2.}

and

$$
\left\langle\varrho_{A}\right\rangle_{H, h, A,-} \leqq\left\langle\varrho_{A}\right\rangle_{H, h, A, b_{A}} \leqq\left\langle\varrho_{A}\right\rangle_{H, h, A,+},
$$

$0 \leqq\left\langle\varrho_{A}\right\rangle_{H, h, \Lambda,+}-\left\langle\varrho_{A}\right\rangle_{H, h, \Lambda,-} \leqq \sum_{p \in A}\left[\left\langle\varrho_{p}\right\rangle_{H, h, A,+}-\left\langle\varrho_{p}\right\rangle_{H, h, \Lambda,-}\right]$ 
The corresponding inequalities for the infinite volume limits to be referred to as $\left(6^{\prime}\right)$ and $\left(7^{\prime}\right)$ are thus also valid.

Proof. (6) follows directly from the F.G.K. result because the field acting on any spin in $\Lambda$ caused by a fixed configuration outside $\Lambda$ lies between that caused by the boundary condition - and by the boundary condition + . (7) follows by observing that the F.G.K. inequalities apply to the function $f_{A}=\sum_{p \in A} \varrho_{p}-\varrho_{A}$, which is increasing in the $\varrho_{p}$.

Lemma 3. $\frac{\partial F(H, h)}{\partial h}$ exists if and only if $\left\langle\sigma_{p}\right\rangle_{H, h,+}=\left\langle\sigma_{p}\right\rangle_{H, h,-}$.

Proof. The assertion will follow if we prove that

$$
\frac{\partial F(H, h)}{\partial h^{ \pm}}=\lim _{\Lambda \rightarrow \infty} \frac{1}{|\Lambda|} \sum_{p \in A}\left\langle\sigma_{p}\right\rangle_{H, h, \pm}=\frac{1}{|\Gamma|} \sum_{p \in \Gamma}\left\langle\sigma_{p}\right\rangle_{H, h, \pm} \equiv \overline{\left\langle\sigma_{p}\right\rangle_{H, h, \pm}}
$$

where $\Gamma$ is the "unit cell" of $H_{p}$ and $|\Gamma|$ is the number of points in $\Gamma$, because by Lemma 2 we know that $\left\langle\sigma_{p}\right\rangle_{H, h,+} \geqq\left\langle\sigma_{p}\right\rangle_{H . h,-}$, so (8) implies that $\left\langle\sigma_{p}\right\rangle_{H, h,+}=\left\langle\sigma_{p}\right\rangle_{H, h,-}$ if $\frac{\partial F(H, h)}{\partial h^{+}}=\frac{\partial F(H, h)}{\partial h^{-}}$and conversely. To prove (8) we first show that the right hand side is equal to $\lim _{\Lambda \rightarrow \infty} m(H, h, \Lambda, \pm)$. Consider first the boundary condition + . For any $\varepsilon>0$ let $\Lambda_{\varepsilon}$ be a box containing $\Gamma$ such that $\left\langle\sigma_{p}\right\rangle_{H, h, \Lambda_{\varepsilon},+} \leqq\left\langle\sigma_{p}\right\rangle_{H, h,+}+\varepsilon$ for all $p \in \Gamma$. Then for any $g \in G$ and $\Lambda$ such that $\Lambda_{\varepsilon}+g \subseteq \Lambda$ we have for all $p \in \Gamma$ :

$$
\begin{aligned}
\left\langle\sigma_{p}\right\rangle_{H, h,+} & =\left\langle\sigma_{p+g}\right\rangle_{H, h,+} \leqq\left\langle\sigma_{p+g}\right\rangle_{H, h, \Lambda,+} \leqq\left\langle\sigma_{p+g}\right\rangle_{H, h, A_{\varepsilon}+g,+} \\
& =\left\langle\sigma_{p}\right\rangle_{H, h, \Lambda_{\varepsilon},+} \leqq\left\langle\sigma_{p}\right\rangle_{H, h,+}+\varepsilon .
\end{aligned}
$$

Using the decomposition $m(H, h, \Lambda,+)=\frac{1}{|\Lambda|} \sum_{p \in \Gamma} \sum_{\substack{p+g \in \Lambda \\ g \in G}}\left\langle\sigma_{p+g}\right\rangle_{H, h, \Lambda,+}$ and the van Hove condition (9) implies that $\overline{\left\langle\sigma_{p}\right\rangle_{H, h,+}} \leqq \varliminf_{\bar{A} \rightarrow \infty} m(H, h, A,+)$ $\leqq \varlimsup_{A \rightarrow \infty} m(H, h, \Lambda,+) \leqq\left\langle\bar{\sigma}_{p}\right\rangle_{H, h,+}+\varepsilon$ for any $\varepsilon$, which means that $\lim _{\Lambda \rightarrow \infty} m(H, h, \Lambda,+)={\overline{\left\langle\sigma_{p}\right.}}_{H, h,+}$, and similarly for the boundary condition - . As mentioned above $\frac{\partial F(H, h)}{\partial h^{+}}$is right continuous and $\frac{\partial F(H, h)}{\partial h}$ exists and is equal to $\lim _{\lambda \rightarrow \infty} m(H, h, \Lambda, b)$ except at most at a denumerable set of points. Hence $\frac{\partial F(H, h)}{\partial h^{+}}=\lim _{h_{n} \rightarrow h_{+}} \frac{\partial F\left(H, h_{n}\right)}{\partial h}=\lim _{h_{n} \rightarrow h_{+}}\left\langle\sigma_{p}\right\rangle_{H, h_{n},+}$ for some sequence $h_{n} \rightarrow h+$. But by Lemma $1\left\langle\sigma_{p}\right\rangle_{H, h_{n},+} \rightarrow\left\langle\sigma_{p}\right\rangle_{H, h,+}$, so (8) is proved for the boundary condition $+;-$ is treated analogously. 
The proof of Theorem 1 now follows directly from the lemmas: By Lemma $1\left\langle\varrho_{A}\right\rangle_{H, h, \pm}$ exist. By Lemma 3 the right side $\left(7^{\prime}\right)$ vanishes if and only if $\frac{\partial F(H, h)}{\partial h}$ exists. When the right side of $\left(7^{\prime}\right)$ vanishes then by $\left(6^{\prime}\right)\left\langle\varrho_{A}\right\rangle_{H, h, b}$ is unique and has the same symmetry as the Hamiltonian. If the right side of $\left(7^{\prime}\right)$ does not vanish there are at least two states with the same symmetry as that of the Hamiltonian.

Theorem 2 follows from Theorem 1 because by Lemma $1 m^{*}=\left\langle\sigma_{p}\right\rangle_{0,0,+}$ and by symmetry $-m^{*}=\left\langle\sigma_{p}\right\rangle_{0,0,-}$, so they are equal if and only if $m^{*}=0$.

Theorem 3 follows in an obvious way from Theorem 1 by the transformation $\hat{\sigma}_{p}=-\sigma_{p}$ if $p \in L_{0}, \hat{\sigma}_{p}=\sigma_{p}$ of $p \in L_{1}$ which transforms the staggered magnetization of the anti-ferromagnet into the magnetization of the ferromagnet.

\section{Concluding Remarks}

(i) The uniqueness of the equilibrium state for a ferromagnet with interactions $J_{p-q} \geqq 0, H=0, h \neq 0$ was first proven by Ruelle [11] using arguments similar to those used in this paper. What we have done is to show, with the help of F.G.K., that for a ferromagnet with pair interactions and with $H_{p}$ periodic and $h$ uniform the continuity of $\frac{\partial F(H, h)}{\partial h}$ is sufficient (and necessary) for the existence of a unique equilibrium state. (The inclusion of $H_{p}$ has further permitted us to extend this result also to anti-ferromagnets.)

(ii) The correlation functions $\left\langle\sigma_{A}\right\rangle_{0, h} h \neq 0$ are also analytic in $h$ and real analytic in the temperature $T$ since the $\left\langle\sigma_{A}\right\rangle_{0, h,+}$ were proven to have this property, Lebowitz and Penrose [7].

(iii) We note that our results are also valid for a system of spins $\sigma_{p}$ taking values $-S,-S+2, \ldots S$ for $S>1$ which interact with a ferroor anti-ferromagnetic pair interaction as in (1). This is true because the F.G.K. inequalities are still valid for such a system if the $\varrho_{p}$ are defined by $\varrho_{p}=\frac{1}{2}\left(\sigma_{p}+S\right)$ [2], and also the Lee-Yang Theorem holds [4]. One only has to consider the functions $\prod_{p \in A}\left(\varrho_{p}\right)^{n_{p}}$ and $C_{A} \sum_{p \in A} \varrho_{p}-\prod_{p \in A}\left(\varrho_{p}\right)^{n_{p}}$ with arbitrary integers $0 \leqq n_{p} \leqq S$ and $C_{A}$ large enough instead of $\varrho_{A}$ and $f_{A}$ used above.

(iv) We remark in connection with Theorem 2 that the uniqueness and translation invariance of the equilibrium state implies that it is ergodic and hence has the clustering property: $\left\langle\sigma_{A+a} \sigma_{B}\right\rangle \rightarrow\left\langle\sigma_{A}\right\rangle\left\langle\sigma_{B}\right\rangle$ in the Cesaro sense as $|a| \rightarrow \infty$ [10]. This implies in particular that there is no long range order when $m^{*}=0$. 
(v) It follows from the Griffiths' inequalities [4] that $m^{*}(T)$ is a non-increasing function of the temperature. Hence $T_{c}$ is uniquely defined by the conditions: $m^{*}(T)=0$ for $T>T_{c}, m^{*}(T)>0$ for $T<T_{c}$. It is known furthermore that at high enough temperatures $m^{*}(T)=0$ and that for many systems, e.g. all two or higher dimensional ones, $m^{*}(T)>0$ at low enough temperatures ${ }^{2}$. Hence for these systems $0<T_{c}<\infty$. The value of $m^{*}\left(T_{c}\right)$ is not known in general (it can, however, be shown that $m^{*}\left(T_{c}\right)=\lim _{\varepsilon \rightarrow 0+} m^{*}\left(T_{c}-\varepsilon\right)$, Lebowitz [8]). It follows from our theorems that if $m^{*}\left(T_{c}\right)=0$ then the correlation functions at $T_{c}$ are independent of the boundary conditions and cluster.

Acknowledgements. This work was started while the authors were attending the 1971 Battelle Summer Rencontres in Seattle directed by A. Lenard. We benefitted much from discussions with our colleagues G. Gallavotti, J. Ginibre, R. Griffiths, O. Lanford and E. Lieb, to all of whom many thanks.

\title{
References
}

1. Dobrushin, R. L.: Funct. Anal. Appl. 2, 292, 302 (1968).

2. Fortuin, C. M., Ginibre, J., Kasteleyn, P.W.: Commun. math. Phys. 22, 89 (1971).

3. Griffiths, R. B.: J. Math. Phys. 8, 478, 489 (1967).

4. - J. Math. Phys. 10, 1559 (1969).

5. - Rigorous results and theorems (preprint).

6. Lanford, O., Ruelle, D.: Commun. math. Phys. 13, 194 (1969).

7. Lebowitz, J. L., Penrose, O.: Commun. math. Phys. 11, 99 (1968).

8. -- More inequalities for Ising ferromagnets (to appear).

9. Lee, T.D., Yang, C. N.: Phys. Rev. 87, 410 (1952).

10. Ruelle, D.: Statistical mechanics. New York: Benjamin 1969.

11. - On the use of "small externals fields" in the problem of symmetry breakdown in statistical mechanics, to appear in Commun. math. Phys.

\author{
A. Martin-Löf's present address \\ Department of Mathematics \\ Royal Institute of Technology \\ Stockholm 70, Sweden
}

${ }^{2}$ These questions are extensively discussed in Ruelle [9] and in Griffiths [6]. 